\title{
Genetic expression and functional characterization of the RUNX2 gene in human adult bone marrow mesenchymal stem cells
}

\author{
J.Y. Zhang ${ }^{1}$ and L.C. Li ${ }^{2}$ \\ ${ }^{1}$ Spine Surgery Department, The People's Hospital of Shouguang, Shouguang, China \\ ${ }^{2}$ Nursing Department, The People's Hospital of Shouguang, Shouguang, China \\ Corresponding author: J.Y. Zhang \\ E-mail: wuzizhengdoc@163.com \\ Genet. Mol. Res. 14 (4): 18210-18217 (2015) \\ Received September 4, 2015 \\ Accepted October 30, 2015 \\ Published December 23, 2015 \\ DOI http://dx.doi.org/10.4238/2015.December.23.8
}

\begin{abstract}
Past studies have revealed the critical role of runt-related transcription factor 2 (RUNX2) in the proliferation and differentiation of mesenchymal stem cells (MSCs). This study therefore aimed to investigate the expression profile of the RUNX2 gene in human bone marrow MSCs and its biological characteristics. Bone marrow MSCs were separated from 12 patients who had received hip joint replacement surgery. After purification and culture, the MSCs were subjected to the 3-(4,5-dimethylthiazol-2yl)-2,5-diphenyltetrazolium bromide assay, flow cytometry, the alkaline phosphatase assay, reverse transcription polymerase chain reaction, and RUNX2 protein quantification. The cell growth curve, staining images, and information on the membrane antigens and the levels of RUNX2 mRNA and protein were obtained based on the results. The growth curve showed, after a 2-day lag period, cultured MSCs entered into the log phase between d3 (Day 3) and d6, when they reached a plateau. Flow cytometry data suggested $94.38 \%$ of MSCs were CD90-positive, while only 3.99 and $1.71 \%$ of total cells were positive for CD35 and CD45, respectively. With
\end{abstract}


the elongated induction period, cultured MSCs were polygonal in shape. After a 14-day induction, cell fusion occurred in the center of the cell nodule accompanied by the disappearance of cellular structure to form the calcium nodule, which was stained red. There was also a statistically significant increase in the level of RUNX2 protein at d7 and d14. An osteogenic medium is required for the differentiation of adult MSCs, which is also under RUNX2 regulation. These findings are potentially valuable for clinical practice.

Key words: Mesenchymal stem cells; Runt-related transcription factor 2; RUNX2; Stem cell differentiation

\section{INTRODUCTION}

Mesenchymal stem cells (MSCs) are adult stem cells that possess the capacity of selfrenewal and the potential for multiple differentiation; they can develop into various cell types including osteocytes, chondrocytes, and adipocytes in a tissue-specific manner (Zhang et al., 2013). Various biological features of MSCs have been described previously, mainly focusing on both the intrinsic and extrinsic conditions that determine their differentiation into different cell types (Hoch et al., 2012). Owing to these prominent features and their ease of transplantation, MSCs have been used in treatments against various diseases including femoral head necrosis, spinal cord injury, immune-related diseases, and myocardial infarction (Chen et al., 2011). As MSCs are stably distributed in the adult body with pluripotent differentiation that is unlimited by cell replication, they have inspiring potential for clinical use within the existing ethical regulation (Gioia et al., 2012). However, as only limited numbers of MSCs exist in a normal adult's bone marrow, and extraction is difficult (Weng and Su, 2013), it is necessary to prepare large numbers of MSCs for clinical use. The specific conditions that are necessary for the induced differentiation of MSCs in human osteoblasts are under the influence of complex intrinsic and extrinsic factors, which results in a high failure rate (Venugopal et al., 2013). As an osteoblast-specific transcription factor, runt-related transcription factor 2 (RUNX2) has cell differentiation facilitation and osteogenic effects. As the current MSCs-related studies mainly focus on high-efficiency separation, purification, and amplification, we aimed to identify a satisfactory method for obtaining large numbers of MSCs. In this study, bone marrow samples were collected from patients in our hospital and then separated and purified. Our objective was to determine the dynamic expression profile of the RUNX2 gene and investigate the biological features of MSCs, in an attempt to find an optimal method for the separation, purification, and amplification of MSCs (Carpenter et al., 2010).

\section{MATERIAL AND METHODS}

\section{General information}

A total of 12 patients who have received hip joint replacement surgery in our hospital between December 2013 and February 2014 were recruited for this study. Bone marrow samples were collected from the proximal femur of each patient. Written consent was obtained from all included patients and their families. The protocol of this study was pre-approved by the Ethical Committee of our hospital. 


\section{Inclusion and exclusion criteria}

All patients received hip joint replacement surgery as a treatment for femoral head necrosis. Patients who were older than 60 years, had a history of chronic infectious disease, mental disorders, or severe systematic diseases were excluded from this study.

\section{Separation, incubation, and purification of MSCs}

Density gradient centrifugation combined with the adherence method was applied to obtain purified MSCs. In brief, bone marrow samples $(5 \mathrm{~mL})$ were collected in a heparin-containing centrifuge tube, which was centrifuged at $1000 \mathrm{rpm}$ for $10 \mathrm{~min}$. The precipitation fraction at the tube bottom was added to an equal volume of phosphate-buffered saline (PBS), and an equal volume of separation buffer was added (Percoll at $1.073 \mathrm{~g} / \mathrm{mL}$ ) (Sigma-Aldrich, St Louis, MO, USA). The whole mixture was then centrifuged at $2500 \mathrm{rpm}$ for $20 \mathrm{~min}$. The monocyte layer was collected, washed twice in PBS, and re-centrifuged at $1500 \mathrm{rpm}$ for $10 \mathrm{~min}$. The collected cells were then re-suspended in $10 \%$ fetal bovine serum-containing culture medium (HyClone, GE Health Care, Logan, UT, USA) and stored in a $25-\mathrm{mL}$ tube at a cell density of $1.0 \times 105 / \mathrm{mL}$. The cells were incubated in a chamber at $37^{\circ} \mathrm{C}$ and the medium was changed every 3 or 4 days, leaving only the adherent cells. When the cell confluence of adherent cells reached $80-90 \%$, the cells were digested with $0.25 \%$ trypsin (HyClone) and $0.02 \%$ ethylenediaminetetraacetic acid, centrifuged, and re-suspended. Quantified cells $\left(1 \times 10^{4} / \mathrm{mL}\right)$ were inoculated into a $20-\mathrm{mL}$ bottle for serial subculture.

\section{3-(4,5-dimethylthiazol-2-yl)-2,5-diphenyltetrazolium bromide (MTT) assay for detecting MSC proliferation}

After three passages, cells were digested and re-suspended as described above to produce cell suspensions of $5 \times 10^{5} \mathrm{~mL}$ density. The MTT assay was applied using an enzymelabeling instrument to plot the growth curve (Luo et al., 2012). The proliferation cycle was analyzed by flow cytometry (BD FACSCalibur, USA). The proliferation index was used to evaluate the level of MSC proliferation.

\section{Cell surface antigen expression assay}

After digestion, centrifugation, and collection, the MSCs were washed in PBS and separated into two tubes; a homotype control was added to one tube, and different fluorescencelabeled primary antibodies (CD34, CD45, CD90 monoclonal antibodies; BD FACSCalibur) were added to the other. Flow cytometry was applied to reveal the surface antigen.

\section{Osteogenic differentiation and alkaline phosphatase assay}

MSCs of the 3rd passage were seeded into 6-well plates, of which two wells were control groups, while the other four wells were induction groups. When cell confluence reached $80 \%$, the MSCs in the induction groups were cultivated using a medium containing human MSC osteogenic differentiation buffer, while the control groups used the intact medium. After 14 days of incubation, the cells were stained to reveal the activity of alkaline phosphatase (Wang et al., 2012). 


\section{Reverse transcription polymerase chain reaction (RT-PCR) for RUNX2 mRNA levels}

MSCs of the 3rd passage were cultivated in osteogenic conditions as described above. The expression level of the RUNX2 gene was detected using RT-PCR at d7 (Day 7), d14, and d21. The primer sequences used are listed in Table 1. The PCR conditions were: pre-denaturation at $95^{\circ} \mathrm{C}$ for $4 \mathrm{~min}$, followed by 45 cycles of denaturation at $95^{\circ} \mathrm{C}$ for $20 \mathrm{~s}, 55^{\circ} \mathrm{C}$ annealing for $15 \mathrm{~s}$, and $72^{\circ} \mathrm{C}$ elongation for $30 \mathrm{~s}$. Cycle threshold (CT) values were calculated based on the RT-PCR results.

Table 1. Primer sequences in reverse transcription polymerase chain reaction (RT-PCR).

\begin{tabular}{ll}
\hline RUNX2-F & 5'-GGACG AGGCA AGAGT TTCAC-3' \\
RUNX2-R & 5'-GAGGG GGTCA GAGAA CAAAC-3' \\
$\beta$-actin-F & 5'-TGCTG ACCGT ATGCA GAAAG-3' \\
$\beta$-actin-R & 5'-GCCAC CAATCC AGACA GAGT-3' \\
\hline
\end{tabular}

\section{RUNX2 protein assay}

Cells were collected at the same time points as those described in the RT-PCR assay. In brief, extracted proteins were separated by gel electrophoresis and transferred to a membrane, which was blocked, then incubated with anti-RUNX2 antibody (Cell Signaling Technology, USA) at $4^{\circ} \mathrm{C}$ overnight, followed by horseradish peroxidase (HRP)-labeled goat anti-rabbit secondary antibody (Santa Cruz Biotechnology, USA). The membrane was then washed, exposed, and developed using chromogenic substrates (Thermo Scientific, USA) followed by imaging analysis using a software package (Clinx Science Instruments, China).

\section{RESULTS}

\section{Morphology of cultured MSCs}

After $24 \mathrm{~h}$ of primary culture, the MSCs showed an adherence growth pattern characterized by polygonal or spindle shapes. At approximately $48 \mathrm{~h}$ there were cell colonies, which broadened and fused to form a monolayer after 7 days. The cell growth rate after the first passage was significantly accelerated, as almost all cells adhered to the wall with few cell deaths within $12 \mathrm{~h}$. The average cell size also increased with more passages.

\section{Proliferation activity of MSCs}

The growth curve of the adult MSCs is presented in Figure 1, which shows an approximate S-curve. After a lag period over the first 2 days in which only a few cells proliferated, the MSCs entered the log phase at $\mathrm{d} 3$ with a positive slope in the growth curve. The cells reached the peak of growth at d6, followed by a plateau period with decreasing increments of cell numbers.

\section{Cell surface antigen assays}

The flow cytometry results showed a positive rate of CD90 in MSCs as high as $94.38 \%$, while only 3.99 and $1.71 \%$ of all MSCs were positive for CD34 and CD45, respectively (Figure 2). 
These results suggest adequate numbers of MSCs in the bone marrow of the proximal femur, and better separation and purification performance using density gradient centrifugation combined with adherence.

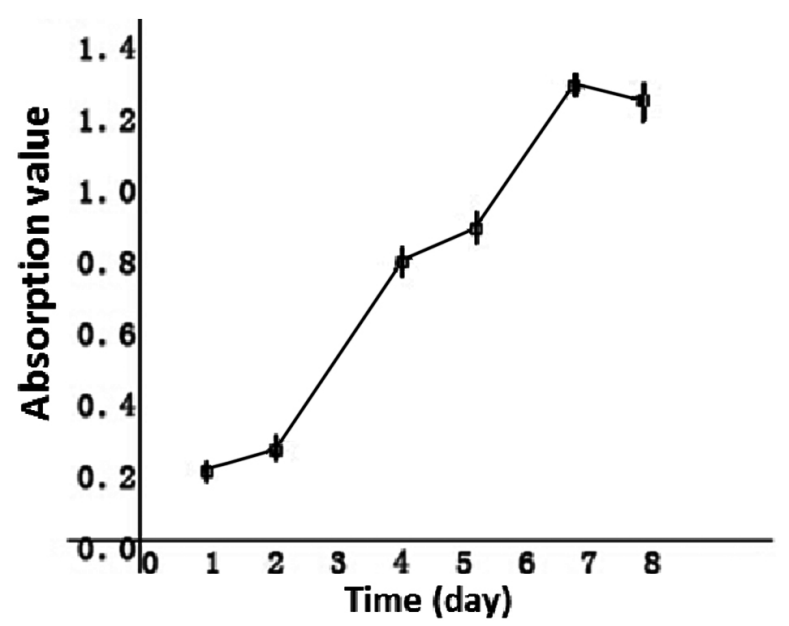

Figure 1. Growth curve of cultured MSCs.
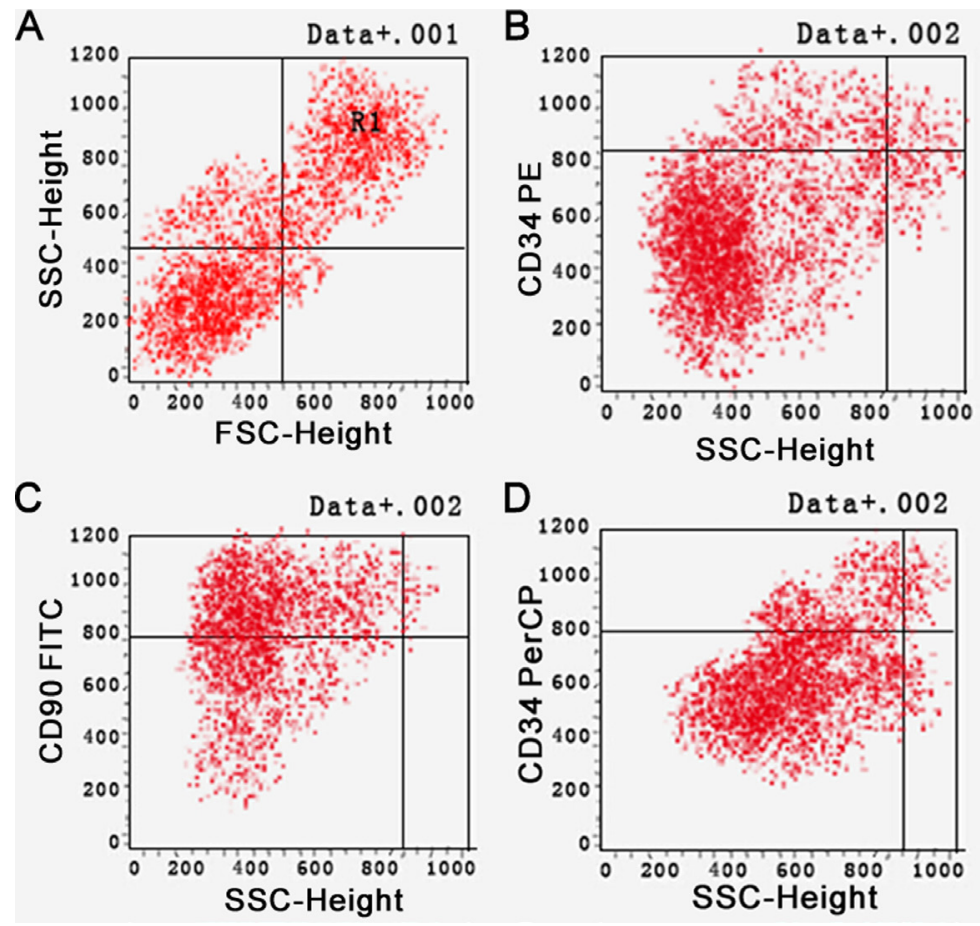

Figure 2. Flow cytometr results of MSCs surface antigen. A. The homogenity test of MSCs. B. CD34 was negatively expressed in most MSCs. C. Most MSCs were CD90-positive. D. CD45 was negative for MSCs. 


\section{Induced differentiation and staining results}

The polygonal shape of the MSCs occurred after continuous induction of differentiation. At d14, cells at the nodular center fused and lost cellular structure to form a calcium nodule, which was stained red, as shown in Figure 3.

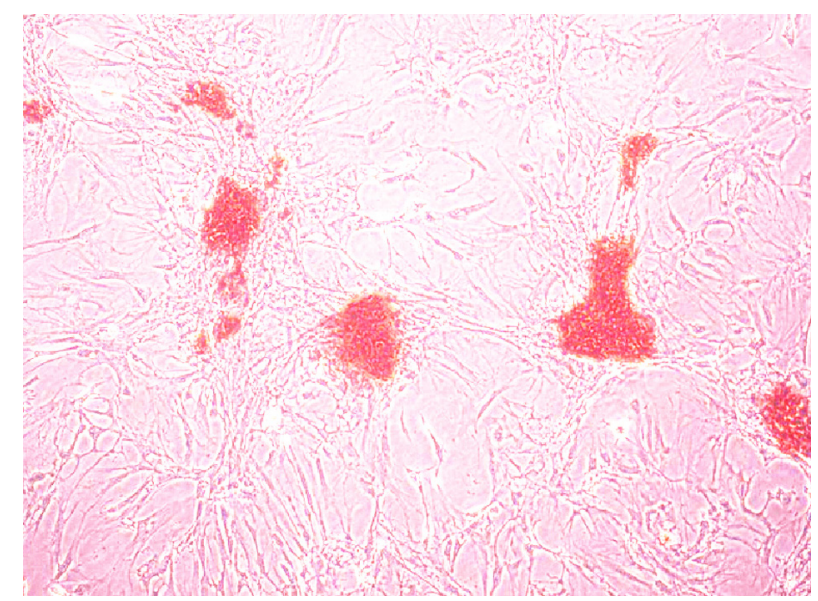

Figure 3. Calcium nodules staining after 14 days of osteogenic induction.

\section{Level of RUNX2 mRNA in MSCs}

In both the control and induction groups, the RUNX2 gene was expressed in the MSCs. In the induction group, MSCs expressed increasing levels of the RUNX2 gene at d7, d14, and d21 after induction (Figure 4), with significant differences compared with the control group $(P<0.05)$. A further comparison revealed that the RUNX2 gene expression level was significantly elevated at d14 compared with d7 $(P<0.05)$, but did not increase significantly from d14 to d21 $(P>0.05)$.

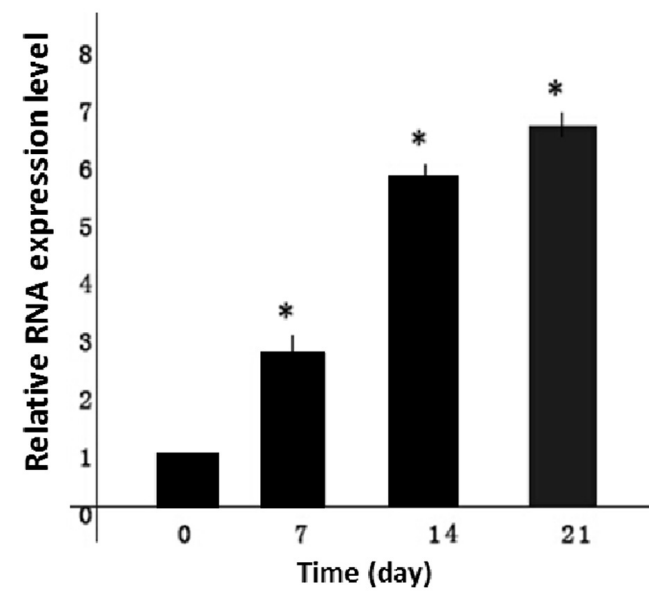

Figure 4. RUNX2 mRNA levels in MSCs after induction. ${ }^{*} \mathrm{P}<0.05$ compared to those in the control group at the same time point. 


\section{Levels of RUNX2 protein in MSCs}

A western blotting assay showed significantly higher expression of RUNX2 proteins in the induction group cells after 7 days of induction (Figure 5). The protein level was also significantly elevated at d14 compared with $d 7(P<0.05)$. However, no significant difference in protein levels was detected between $\mathrm{d} 21$ and $\mathrm{d} 14$.

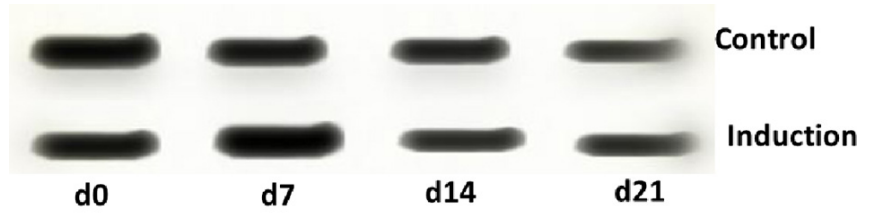

Figure 5. RUNX2 protein expression levels by Western blotting.

\section{DISCUSSION}

Originating in the mesoderm layer, MSCs are non-hematopoietic stem cells that do not express blood cell surface antigens, but only the surface markers of stromal and interstitial cells, such as CD44, CD73, CD90, and CD105 (Baksh et al., 2004; Liu et al., 2013). This study revealed that $94.38 \%$ of the MSCs were CD90-positive, while only $3.99 \%$ were CD34-positive and only $1.71 \%$ were CD45-positive, in agreement with a previous study (Tyndall, 2012). However, as no cell-specific surface antigen exits for MSCs, it is necessary to label them with surface antigens to evaluate cell purity and homogeneity (Forostyak et al., 2013; Tan et al., 2013). This study utilized a classical method for the induction of osteogenic differentiation, leading to the precipitation of calcium salts and mineralized nodules, which were all positively stained, on the surface of the MSCs at d14 and d21 after induction. The differentiation of MSCs is dependent on several conditions, such as the osteogenic induction factor used in this study, in which the application of an MSC osteogenic induction solution can stimulate the differentiation of MSCs into osteocytes. The positive staining revealed the MSCs' inherent ability to proliferate for bone formation, which is a biological characteristic of MSCs (Waterman et al., 2010; Glass et al., 2011; Nam et al., 2012). This study observed significantly elevated levels of both RUNX2 mRNA and proteins at d7, d14, and d21 after induction, indicating the potentially important role of RUNX2 in bone formation, although the detailed mechanism still requires further research.

In summary, in this study we performed the entire process of extraction, cultivation, purification, and induction of MSCs from bone marrow. We also monitored RUNX2 expression levels and the biological features of bone marrow MSCs. Using the method of whole bone marrow density gradient centrifugation combined with adherence, we successfully obtained and cultivated adult bone marrow MSCs with higher purity using a simple procedure (Lepperdinger, 2011). This strategy, therefore, could be used to obtain large numbers of MSCs for treatment purposes (Yoon et al., 2011; Tang et al., 2012). Particular induction reagents and culture conditions should be used for MSC cultivation to satisfy the requirements of different diseases. This study provides both basic knowledge and practical guidance for clinical treatment.

\section{Conflicts of interest}

The authors declare no conflict of interest. 


\section{ACKNOWLEDGMENTS}

Thank you to all the authors.

\section{REFERENCES}

Baksh D, Song L and Tuan RS (2004). Adult mesenchymal stem cells: characterization, differentiation, and application in cell and gene therapy. J. Cell Mol. Med. 8: 301-316.

Carpenter RS, Goodrich LR, Frisbie DD, Kisiday JD, et al. (2010). Osteoblastic differentiation of human and equine adult bone marrow-derived mesenchymal stem cells when BMP-2 or BMP-7 homodimer genetic modification is compared to BMP2/7 heterodimer genetic modification in the presence and absence of dexamethasone. J. Orthop. Res. 28: 1330-1337.

Chen Z, Wang X, Shao Y, Shi D, et al. (2011). Synthetic osteogenic growth peptide promotes differentiation of human bone marrow mesenchymal stem cells to osteoblasts via RhoA/ROCK pathway. Mol. Cell Biochem. 358: 221-227.

Forostyak S, Jendelova P and Sykova E (2013). The role of mesenchymal stromal cells in spinal cord injury, regenerative medicine and possible clinical applications. Biochimie 95: 2257-2270.

Gioia R, Panaroni C, Besio R, Palladini G, et al. (2012). Impaired osteoblastogenesis in a murine model of dominant osteogenesis imperfecta: a new target for osteogenesis imperfecta pharmacological therapy. Stem Cells 30: 1465-1476.

Glass GE, Chan JK, Freidin A, Feldmann M, et al. (2011). TNF-alpha promotes fracture repair by augmenting the recruitment and differentiation of muscle-derived stromal cells. Proc. Natl. Acad. Sci. U. S. A. 108: 1585-1590.

Hoch Al, Binder BY, Genetos DC, Leach JK, et al. (2012). Differentiation-dependent secretion of proangiogenic factors by mesenchymal stem cells. PLoS One 7: e35579.

Lepperdinger G (2011). Inflammation and mesenchymal stem cell aging. Curr. Opin. Immunol. 23: 518-524.

Liu H, Peng H, Wu Y, Zhang C, et al. (2013). The promotion of bone regeneration by nanofibrous hydroxyapatite/chitosan scaffolds by effects on integrin-BMP/Smad signaling pathway in BMSCs. Biomaterials 34: 4404-4417.

Luo F, Hou T, Zhang Z, Xie Z, et al. (2012). Effects of pulsed electromagnetic field frequencies on the osteogenic differentiation of human mesenchymal stem cells. Orthopedics 35: e526-e531.

Nam D, Mau E, Wang Y, Wright D, et al. (2012). T-lymphocytes enable osteoblast maturation via IL-17F during the early phase of fracture repair. PLoS One 7: e40044.

Tan SL, Ahmad TS, Selvaratnam L, Kamarul T, et al. (2013). Isolation, characterization and the multi-lineage differentiation potential of rabbit bone marrow-derived mesenchymal stem cells. J. Anat. 222: 437-450.

Tang QL, Xie XB, Wang J, Chen Q, et al. (2012). Glycogen synthase kinase-3 $\beta$, NF-kB signaling, and tumorigenesis of human osteosarcoma. J. Natl. Cancer Inst. 104: 749-763.

Tyndall A (2012). Application of autologous stem cell transplantation in various adult and pediatric rheumatic diseases. Pediatr. Res. 71: 433-438.

Venugopal J, Rajeswari R, Shayanti M, Low S, et al. (2013). Electrosprayed hydroxyapatite on polymer nanofibers to differentiate mesenchymal stem cells to osteogenesis. J. Biomater. Sci. Polym. Ed. 24: 170-184.

Wang H, Pang B, Li Y, Zhu D, et al. (2012). Dexamethasone has variable effects on mesenchymal stromal cells. Cytotherapy 14: $423-430$.

Waterman RS, Tomchuck SL, Henkle SL and Betancourt AM (2010). A new mesenchymal stem cell (MSC) paradigm: polarization into a pro-inflammatory MSC1 or an Immunosuppressive MSC2 phenotype. PLoS One 5: e10088.

Weng JJ and Su Y (2013). Nuclear matrix-targeting of the osteogenic factor Runx2 is essential for its recognition and activation of the alkaline phosphatase gene. Biochim. Biophys. Acta 1830: 2839-2852.

Yoon IS, Chung CW, Sung JH, Cho HJ, et al. (2011). Proliferation and chondrogenic differentiation of human adipose-derived mesenchymal stem cells in porous hyaluronic acid scaffold. J. Biosci. Bioeng. 112: 402-408.

Zhang Y, Zhang DY, Zhao YF, Wang J, et al. (2013). Analysis and characterization of the functional TGF $\beta$ receptors required for BMP6-induced osteogenic differentiation of mesenchymal progenitor cells. BMB Rep. 46: 107-112. 